\title{
The C3PO Project: a laser communication system concept for small satellites
}

\author{
Benoît d'Humières* (1) \\ Bruno Esmiller, Yann Gouy, Emilie Steck, ${ }^{(2)}$ \\ Crisanto Quintana, Graham Faulkner, Dominic O’Brien ${ }^{(3)}$ \\ Fabian Sproll, Paul Wagner, Daniel Hampf, Wolfgang Riede ${ }^{(4)}$ \\ Michael Salter, Qin Wang, Duncan Platt, Darius Jakonis, Xiaoyu Piao, Mikael Karlsson, Olof \\ Oberg, Ingemar Petermann ${ }^{(5)}$ \\ Aneta Michalkiewicz, Jerzy Krezel, Anna Debowska ${ }^{(6)}$ \\ Yoann Thueux ${ }^{(7)}$ \\ (1) TEMATYS, 6 cité de Trévise - 75009 PARIS - France \\ (2) Airbus Safran Launchers SAS, 51-61 route de Verneuil, 78133 Les Mureaux Cedex, France \\ (3) Department of Engineering Science, University of Oxford, Parks Road, OX13PJ, UK. \\ (4) Institute of Technical Physics, German Aerospace Center, Pfaffenwaldring 38-40, 70569 \\ Stuttgart, Germany \\ ${ }^{(5)}$ Sensor System Department, RISE Acreo AB, Box 1070, 16440 Kista, Sweden \\ (6) Astri Polska Sp. z o.o., Tamka 3, 00-349 Warszawa, Polska \\ (7) Airbus Group Innovations, Wellington House, 125 Strand, London, WC2R OAP
}

\begin{abstract}
The satellite market is shifting towards smaller (micro and nanosatellites), lowered mass and increased performance platforms. Nanosatellites and picosatellites have been used for a number of new, innovative and unique payloads and missions. This trend requires new concepts for a reduced size, a better performance/weight ratio and a reduction of onboard power consumption. In this context, disruptive technologies, such as laser-optical communication systems, are opening new possibilities.

This paper presents the $\mathrm{C}^{2} \mathrm{PO}^{1}$ system, "advanced Concept for laser uplink/ downlink CommunicCation with sPace Objects", and the first results of the development of its key technologies. This project targets the design of a communications system that uses a ground-based laser to illuminate a satellite, and a Modulating Retro-Reflector (MRR) to return a beam of light modulated by data to the ground. This enables a downlink, without a laser source on the satellite. This architecture suits well to small satellite applications so as high data rates are potentially provided with very low board mass. C3PO project aims to achieve data rates of 1Gbit/s between LEO satellites and Earth with a communication payload mass of less than 1kilogram. In this paper, results of the initial experiments and demonstration of the key technologies will be shown.
\end{abstract}

Keywords: Free space optics, laser communication, microsatellite, CubeSat, ground station, modulating retro-reflector

* bdhumieres@tematys.com; phone +336 746452 21; www.tematys.com

${ }^{1} \mathrm{C} 3 \mathrm{PO}$ is an H2020 project financed by the European Commission with Grant Agreement: 637595 


\section{INTRODUCTION}

This paper presents the C3PO system, "advanced Concept for laser uplink/ downlink CommuniCation with sPace Objects", and the first results of the development of its key technologies.

The C3PO project includes the following activities:

$>$ Design of the system architecture

$>$ Development of the key enabling technologies (Satellite target acquisition, Safety and security, Modulating retro-reflector)

$>$ Demonstration of the system concept through two experiments (acquisition of a satellite target with a laser station and an outdoor communication experiment based on the Modulating retro-reflector)

$>$ Elaboration of innovative business models well suited to the specificities of nanosatellites programs for the commercialization of uplink/downlink communication services based on the concept.

The need for developing nanosatellite Laser communication is widely acknowledged [1]. Current small satellites use RF technology to communicate with the ground station, operating VHF, UHF, S, X, Ku, K or Ka band transceivers. The X and K-band can support more than 100Mbps data rates to CubeSats, with a mass of a few Kgs and an electrical power consumption of a few dozens of watts. To give examples, data rates of $150 \mathrm{Mbps}$ to $4 \mathrm{Gbps}$ can be achieved for terminal masses of 2.3 to $4 \mathrm{~kg}$ and power consumption of 30 to $120 \mathrm{~W}$ [2-4]. Besides the data rate and power consumption in these approaches, the growing congestion of the electromagnetic spectrum has led to free space optics (FSO) to become an alternative to RF systems in implementing long range point to point high speed data links. FSO laser communication systems have been explored extensively in ground-based communication systems, as well as for in-orbit and interplanetary spacecraft missions. These systems can transfer large amounts of data with a significant decrease in power requirements and hardware mass compared to traditional RF band-based communication systems.

On large satellites, an optical link with a data rate of more than $5 \mathrm{Gbps}$ has already been demonstrated [5]: The mass of a typical embedded laser communication system is few dozens of $\mathrm{kg}$ and its electrical consumption is more than $100 \mathrm{~W}$. In June 2016, the satellite BIROS was launched, equipped with the OSIRISv2 laser communication payload, developed by the DLR. Even dedicated to a bigger satellite than a CubeSat, as BIROS weights $115 \mathrm{~kg}$, the optical payload is to demonstrate a data rate of $1 \mathrm{Gbit} / \mathrm{s}$ with a $1 \mathrm{~W}$ laser for a mass of $1,65 \mathrm{~kg}$ and a power consumption of $37 \mathrm{~W} \mathrm{[6].}$

On CubeSats, several laser-based communication systems are under development, all using laser sources on the satellite: the Aerospace Corporation Optical Communication and Sensor Demonstration (OCSD), with an equipment mass of $3 \mathrm{~kg}$ and an electrical consumption of 50W, was launched in 2015, with an expected data rate of 5Mbps [7].

Figure 1 shows a comparison of data rate vs data rate/power for different state of the art space to ground communications systems. This plot includes RF and FSO systems. C3PO provides a very high data rate whilst having the best data rate to power consumption ratio. 


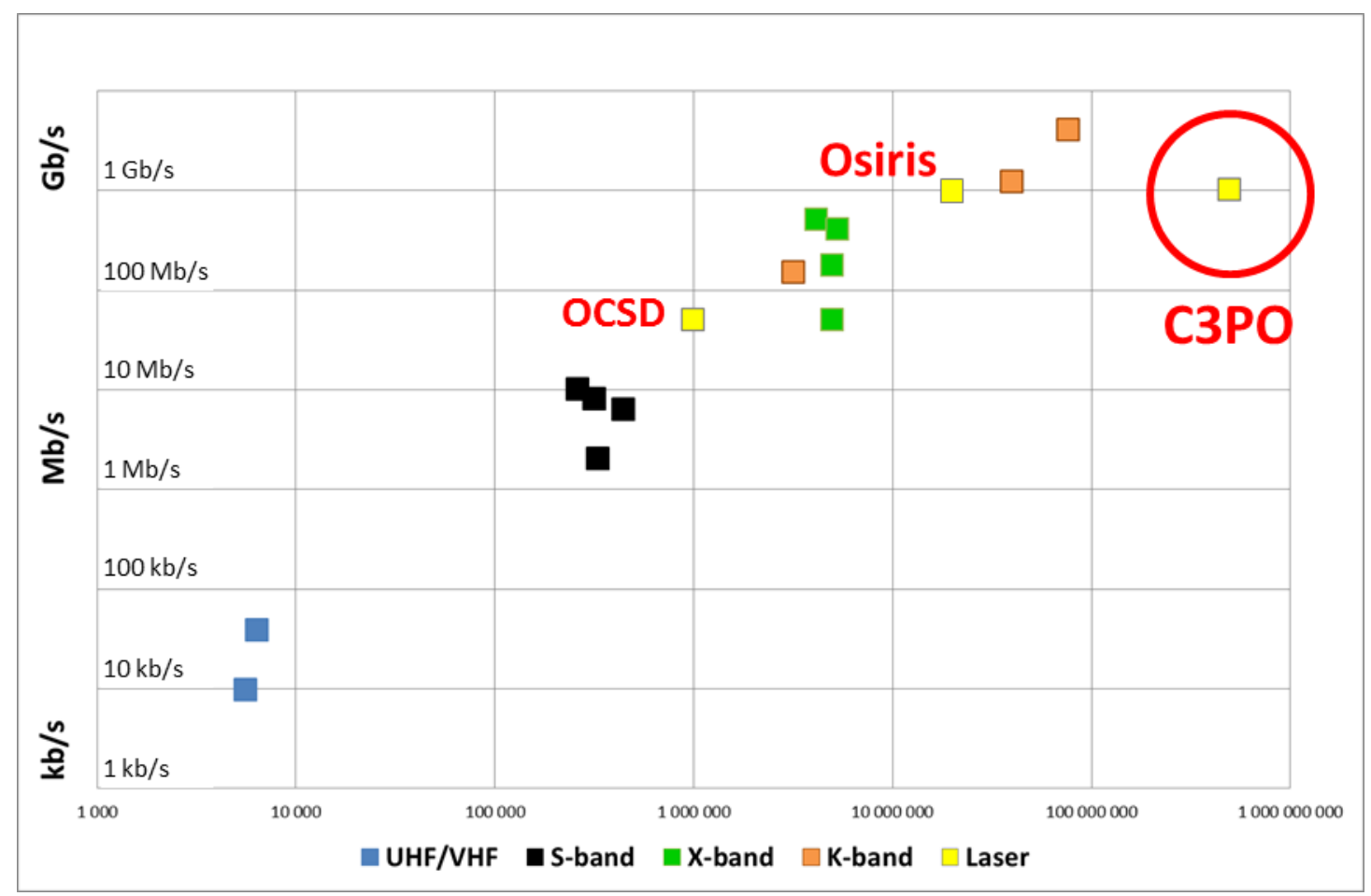

Figure 1: Data rate vs. Data rate/power consumption for different space-to-ground communications technologies.

\section{C3PO CONCEPT AND SYSTEM DESCRIPTION}

The C3PO concept has been designed to provide a full uplink/downlink communication functionalities to nanosatellite programs, particularly those with limited budgets. Figure 2 shows the C3PO system architecture, which is composed by three sub-systems:

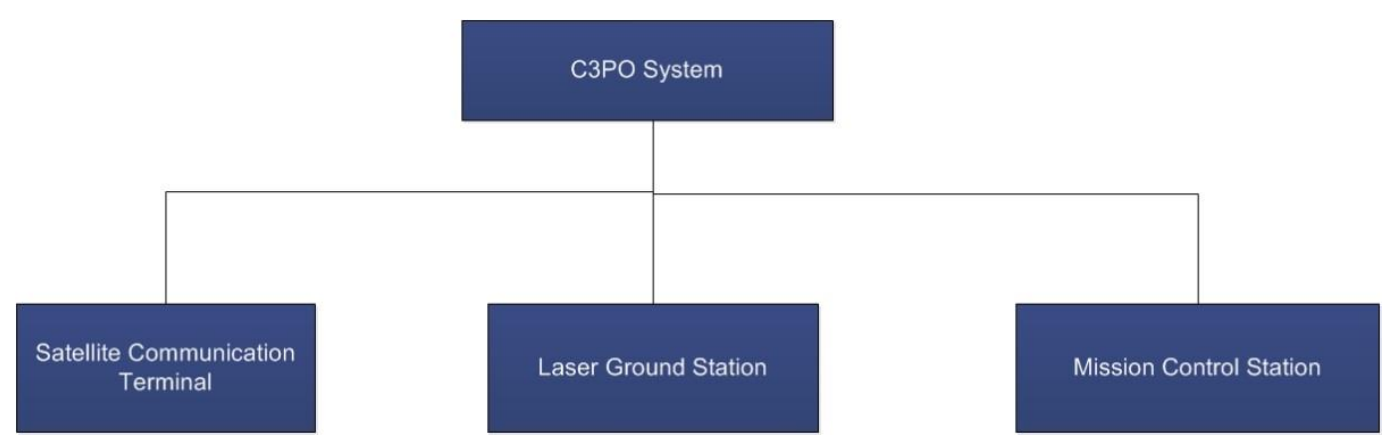

Figure 2: C3PO system architecture concept

The Satellite Communication Terminal (SCT): a lightweight optical communication terminal embedded on the satellite and allowing for laser signal reception, modulation and reflection (the targeted mass is less than $1 \mathrm{~kg}$ and the electrical power consumption lower than $2 \mathrm{~W}$ ). 
$>$ The Laser Ground Station (LGS): a ground-based station that performs the satellite acquisition and pointing, the laser signal emission (modulated for uplink, continuous for downlink) and the laser signal reception.

$>$ The Mission Control Station (MCS): a ground-based station that monitors and controls the set of laser ground stations and dispatches the workload between the available ground stations, in order to acquire all the datasets from the satellites constellation.

Figure 3 a) and b) shows the C3PO system. Each ground-based station is equipped with components used for both uplink and downlink communications: a telescope for satellite acquisition, tracking and receiving the downlink photons, a dedicated transmitter optics for pointing the laser beam towards the satellite and a ground-based laser emitting continuously (for downlink communication) or in a modulated mode (for uplink communication). Downlink communications (figure 3a) will use a space-based lightweight terminal equipped with a modulating retro-reflector (MRR). This terminal reflects the beam from the ground station and modulates it to send data back to the ground station, where a ground-based optical receiver decodes the data. Uplink communications (figure 3b) will use an embedded optical receiver on the satellite, receiving the modulated laser signal from the ground.

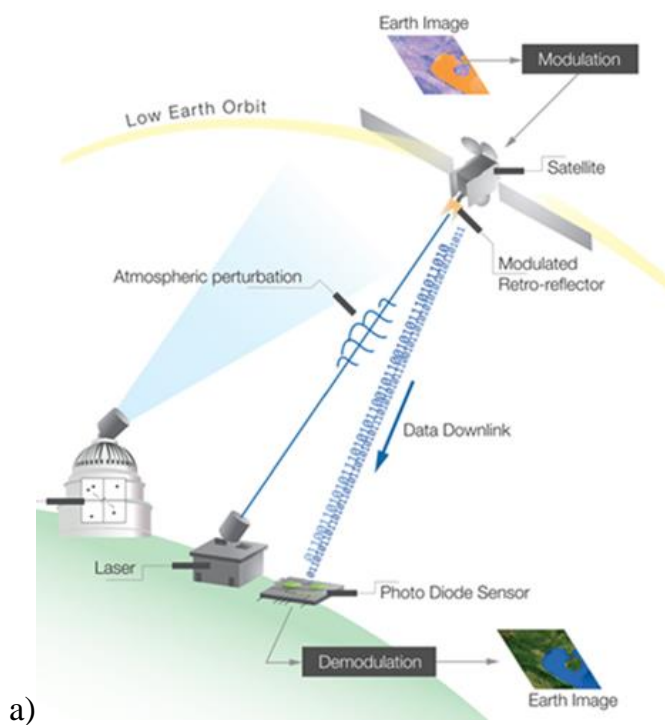

Figure 3: downlink mode concept (a) and uplink mode concept (b)

b)

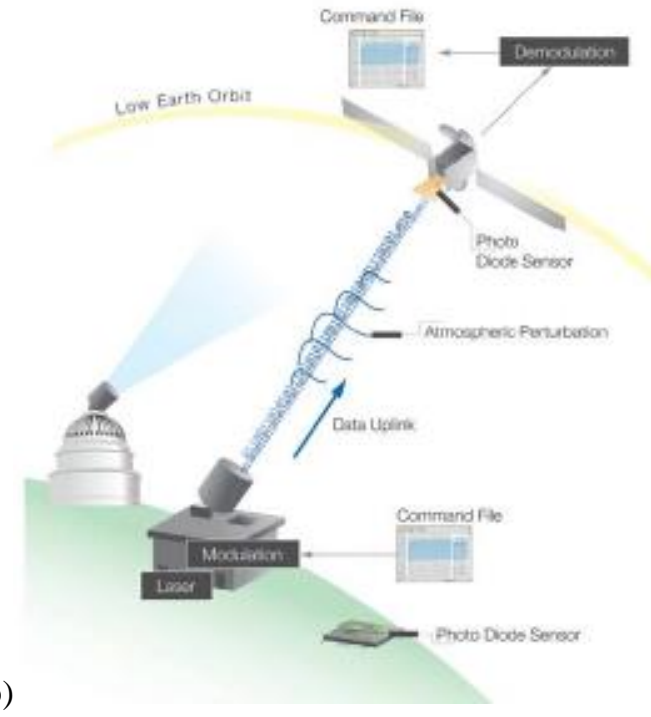

C3PO aims to validate these concepts by developing the core technologies and validating them with two field experiments. In the next sections the MRR and the demonstration experiments are described.

\section{MODULATING RETRO-REFLECTOR}

The modulation principle of the EAM (Electro-Absorption Modulator) is based on the Quantum-Confined Stark effect (QCSE), which describes the effect of an external electric field upon the light absorption spectrum of a Quantum Well (QW). When an electrical field is applied to the QW, two effects appear simultaneously as illustrated in Figure 4. One effect is that the electron and hole-energy levels are moved closer to each other (Stark effect), resulting in an absorption shift to longer wavelengths. Another effect is that the bands tilt and the confined electron and hole related to the exciton are pulled by the electric field in opposite directions, thereby causing the overlap between the electron- and the holewavefunction to decrease, broadening the spectrum of the excitonic peak and decreasing its intensity. This shift in the absorption band-edge of the device under electric field can be used to modulate an optical signal at a wavelength close to 
that edge, by using the field to change the absorption of the material. Based on physical mechanisms behind the electroabsorption modulation we can identify the overall performance of EAMs using their operating bias, contrast ratio, loss, and power consumption as a figure of merit.

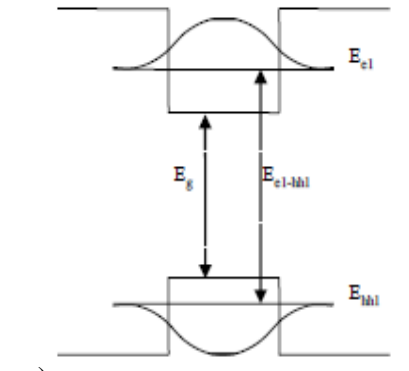

a)

Figure 4: Quantum well under electrical field at zero (a) and reverse bias (b)

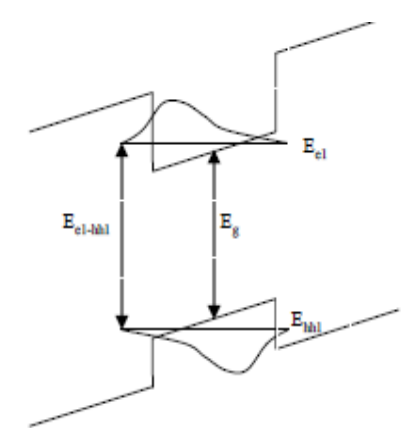

Surface normal EAMs offer significant advantages in terms of polarisation insensitivity, large active apertures and low insertion losses. A drawback of these modulators is the short interaction length between the incident light and the active medium, which limits the contrast ratio. Single-path surface normal EAMs have typical contrast ratios in the range 2:1.

In the C3PO project, 6x6 EAM arrays operating in the infrared C-band (1530-1565 nm) were designed. They are surface normal devices based on $80 \mathrm{InGaAs} / \mathrm{InAlAs}$ coupled quantum wells embedded in a pin diode structure. The EAM can operate in a transmissive or reflective mode that uses a metal mirror coated on the EAM's backside [8]. A reflective mode is being used in this work. The pixel size of the array is $250 \mu \mathrm{m}$ x $250 \mu \mathrm{m}$ with a pitch of $255 \mu \mathrm{m} \times 255 \mu \mathrm{m}$. To drive each pixel individually a wire-bonding pad $(70 \mu \mathrm{m} \times 70 \mu \mathrm{m})$ was arranged on the top of the pixels, which will block some of the incident optical beam. Thus, the fill factor of the EAM array is about $88.5 \%$ including the $5 \mu \mathrm{m}$ gaps between the pixels and the wire-bonding pad on the top of the pixels. One of the fabricated 6x6 arrays mounted on its PCB (printed circuit board) is shown in Figure 5a.
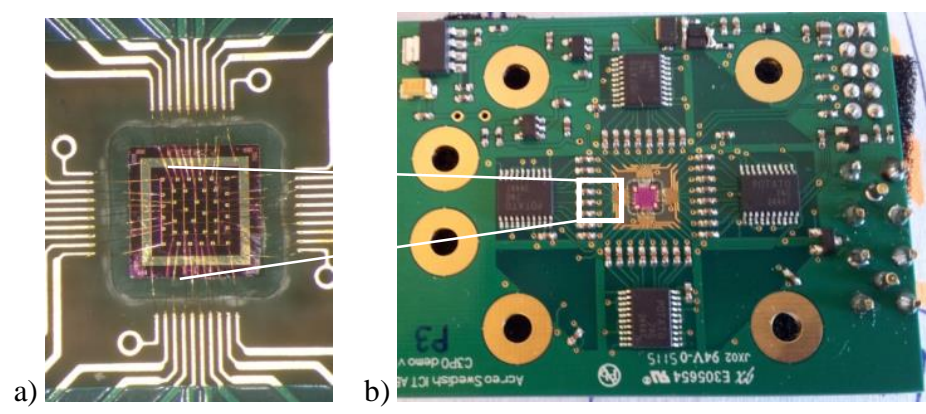

Figure 5: A fabricated 6x6 EAM array electrically connected with its driver electronics by wire-bonding

To verify the absorption properties of the fabricated EAM, their transmittance spectra under different reverse bias up to $6 \mathrm{~V}$ were measured using a Bruker V80 FTIR spectrometer. Figure 6 a and b shows the transmittance and contrast ratio measured at $25^{\circ} \mathrm{C}$. The contrast ratio was derived from the transmittance spectra at different biases divided by its transmittance at zero bias. Values of 1.385 has been achieved at room temperature and using voltages up to $6 \mathrm{~V}$. 

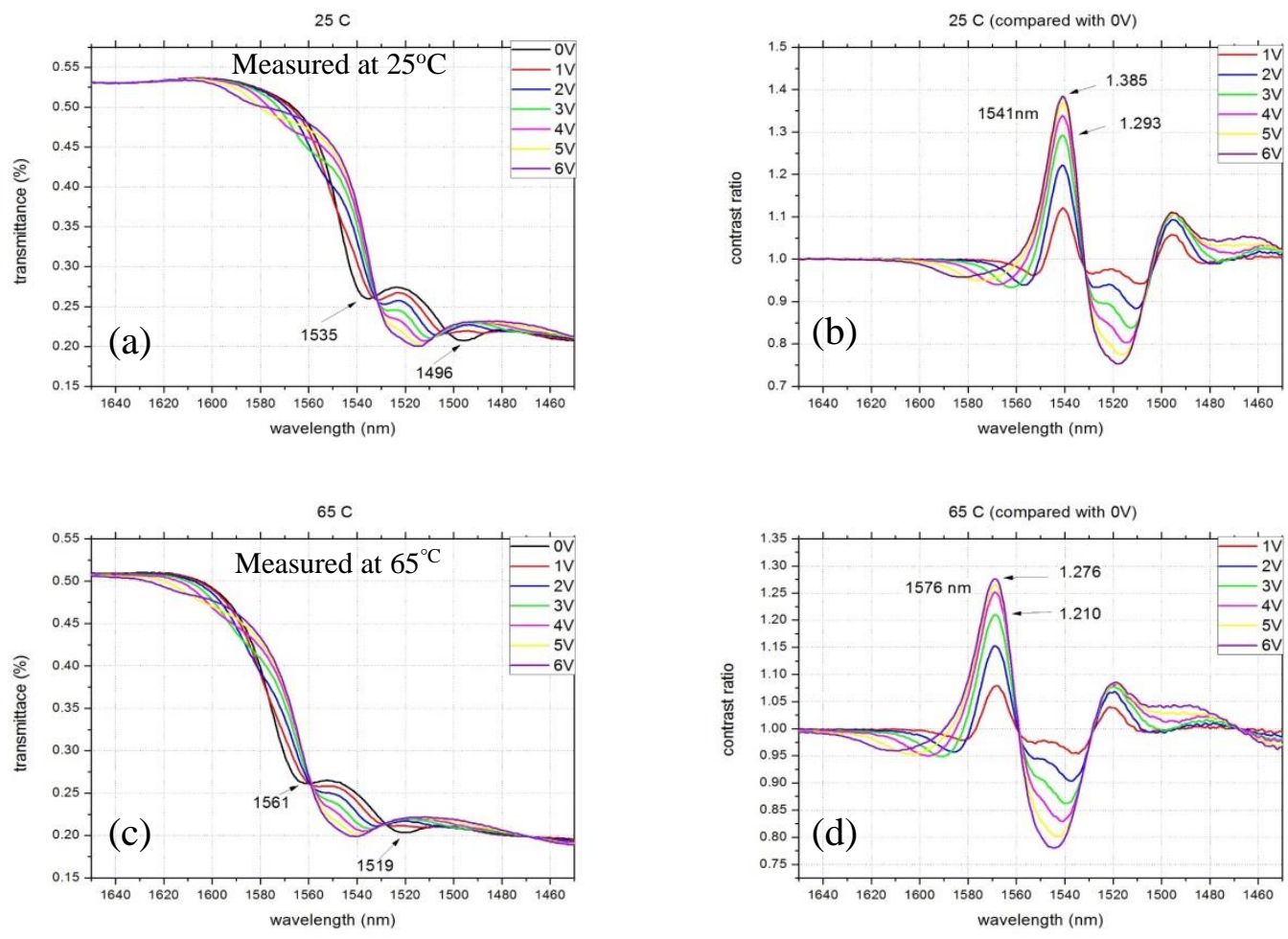

Figure 6: Transmittance spectra and contrast ratio of the EAM array measured by FTIR, at $25^{\circ} \mathrm{C}$ (a and b) and $65^{\circ} \mathrm{C}(\mathrm{c}$ and d), for different biases

The MQW absorption is temperature dependent. To characterize the thermal effect on the EAMs, transmittance spectra were measured for a temperature range from $17^{\circ} \mathrm{C}$ to $70^{\circ} \mathrm{C}$, using a transmissive type EAM. Figure 6 (a) and (c) show the transmittance spectra measured at $25^{\circ} \mathrm{C}$ and $65^{\circ} \mathrm{C}$, respectively, and their corresponding contrast ratios as illustrated in Figure 6 (b) and (d), respectively. The results reveal clear red-shift of the QW absorption peaks (transmittance dips) with increasing temperature, and about $10 \%$ reduction of the contrast ratio at $65^{\circ} \mathrm{C}$ compared with the $25^{\circ} \mathrm{C}$ case. A a shift in operating wavelength at which the maximum contrast ratio of $0,7 \mathrm{~nm} /{ }^{\circ} \mathrm{C}$ has been measured.

Custom driver electronics also fabricated by Acreo using commercially available electronic elements and integrated circuits as shown in the Figure 5b. The board has a single LVDS (Low Voltage Differential Signaling) input, and a binary data input signal is used to apply the same modulated voltage to each pixel. The modulation bandwidth of the EAM array/driver combination was tested by using a square wave as input to the modulator driver electronics. A bandwidth of $150 \mathrm{MHz}$ was measured. Figure 7 shows a $125 \mathrm{MHz}$ square driving signal and the optical modulated signal. This experiment was performed with a $1547 \mathrm{~nm}$ interrogator beam. A reverse voltage bias of $-3.8 \mathrm{~V}$ was applied to the pixels. 


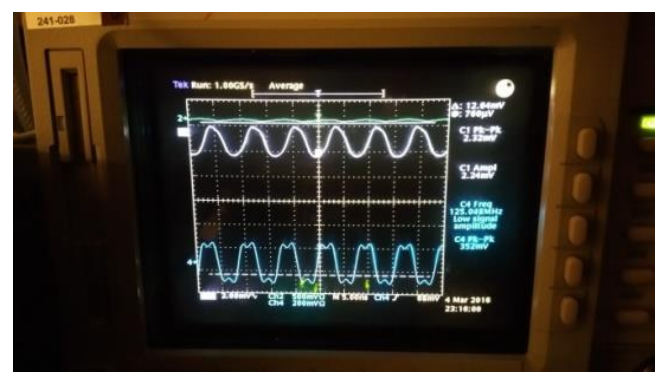

Figure 7; Optical modulation signals of one 6x6 EAM arrays at $125 \mathrm{MHz}$.

The first-generation driver electronics dissipates significant power, leading to a rise in operating temperature over time and then a shift in the contrast ratio peak. Additionally, non-linear distortions caused by unmatched load driving were also observed. This phenomenon is currently limiting the maximum data rate achievable with this MRR. A new version of the driver electronics is currently under development to tackle these issues.

The second-generation driver electronics for MRR V2 is under development. In the new MRR version the LVDS input signal is distributed into different driver channels and then amplified by several stages of microwave amplifiers. To save the power consumption the PHEMT based output stage is configured to drive multiple ( 2 or 4 ) pixels of the EAM array. The MRR V2 is aimed to achieve $1 \mathrm{Gbps}$ data rate $(500 \mathrm{MHz}$ operation frequency).

\section{DESCRIPTION OF EXPERIMENTS}

Two experiments have been defined in C3PO project to validate a set of system parameters and consolidate the system architecture described in section 2. Experiment 1 aims the tracking of a LEO satellite equipped with retro-reflector. This experiment will show not only the tracking capabilities of the developed base station, but also will provide valuable information to assess the link budget. The main contributions from this experiment concern levels of signal and noise, backscattering effects, tracking accuracy and tracking acquisition time. Experiment 2 targets a demonstration of a data link of 1 Gbps between an unmanned aerial vehicle (UAV) and a base station (BS) using a modulating retroreflector (MRR). The targeted link range is $1 \mathrm{~km}$. The main contributions from this experiment concern the MRR requirements (mass, power consumption), field test operability in usual atmospheric conditions (temperature, rain, humidity) and communication concepts (achievability of a high data rate given a certain signal-to-noise ratio). Each of the experiments is described in more detail in the following section.

\section{FIRST EXPERIMENT: ESTIMATION OF THE LINK BUDGET}

In order to estimate the feasibility of the C3PO system a laser link to LEO satellites has been investigated experimentally. Parameters such as atmospheric backscattering, laser link acquisition duration, minimal elevation of successful laser link and achievable laser link time per pass were measured. The experiment was run on the DLR's ground station based in Stuttgart (see Figure 8), containing a Planewave CDK 17" Cassegrain telescope connected to a Andor Zyla 5.5 sCMOS camera on an astronomical mount, a laser transmitter as well as dedicated electronics.

As a first step, the tracking sub-system has been tested in order to confirm that it could provide a sufficient accuracy for detecting and following a LEO satellite through the whole pass. Then, the return ratio (detected pulses over sent laser pulses ratio) were measured and compared to a theoretical model to assess the link loss. 


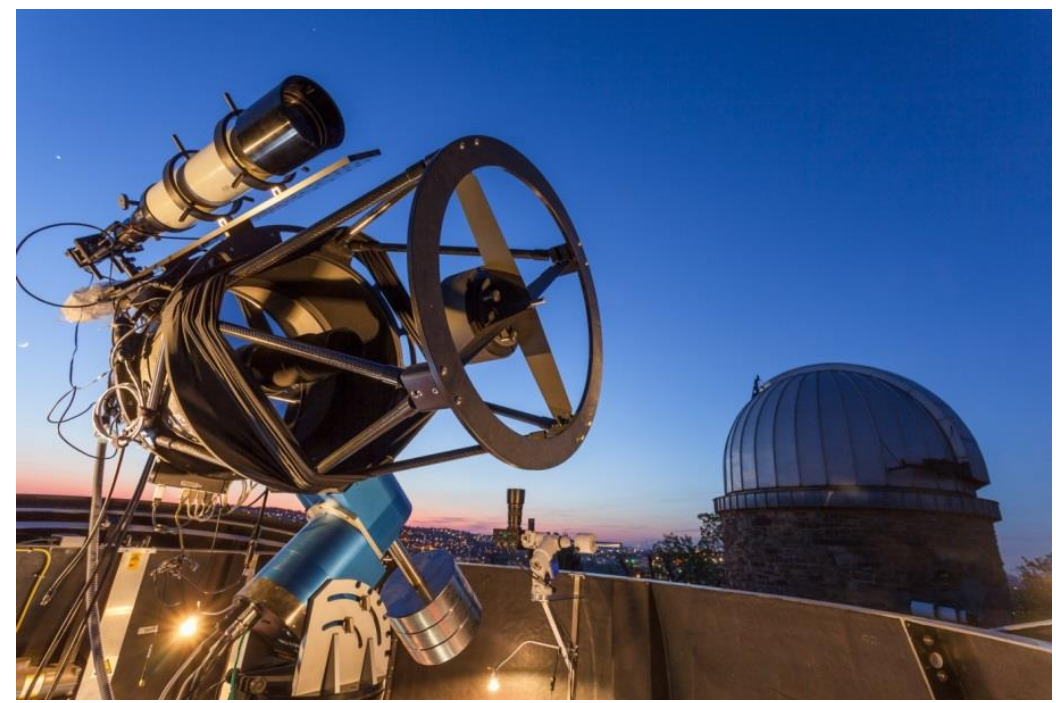

Figure 8: The DLR ground station in Stuttgart, Germany will perform link budget measurements to satellites using a pulsed Nd:YAG laser (1064nm, 3ns, 1-10kHz) as source and an InGaAs based SPAD as detector.

\subsection{Evaluation of the tracking set-up}

Tracking of the target is realized by a coarse pointing of the telescope using the publicly available TLE data and an additional passive optical closed loop system for fine tracking. Reflected sunlight from the satellite imaged onto the sCMOS camera, is used as input variable to the closed loop system. Trajectories of satellites are loaded on a custommade control software in TLE format. The telescope interface calculates the satellite positions including corrections for refraction and the telescope pointing model. The software ties directly into the telescope hardware to move both axes along predefined trajectories. A GPS clock within the telescope control ensures that the trajectory is synchronized exactly to UTC.

The image taken by the camera is analysed directly after the exposure by a dedicated algorithm with the goal to detect the position of the object in the image. All LEO objects (except the ISS) appear as point-like structures in the optical system; however, the point may be distorted by atmospheric turbulences, especially when using short exposure times (one example is shown in Figure 9). 


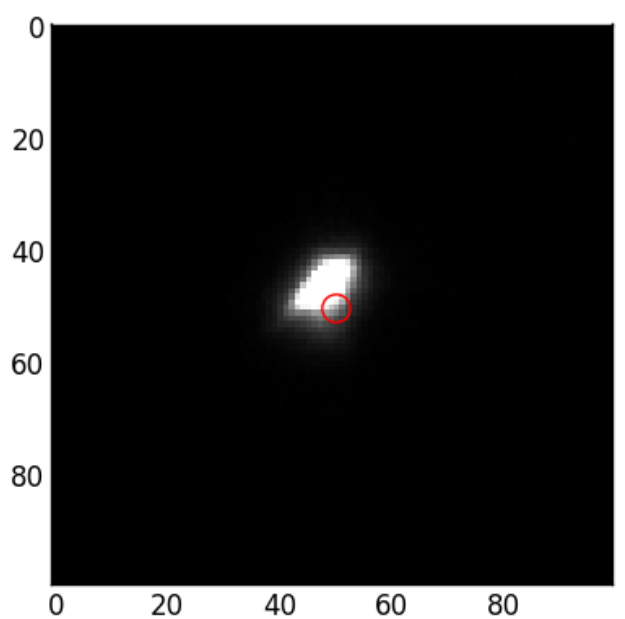

Figure 9: Typical tracking image with a small Area of Interest of $100 \times 100$ pixels to reduce processing time. In this particular image the deviation of the object to the target point (red circle) is about 6 pixels, or 3 arcseconds (2015-1004, 18:45:01 UTC). Atmospheric turbulences cause the object to appear non-spherical.

The software extracts the properties of the clearest structures from the background level. For each of them, size, centre of gravity and eccentricity, are calculated. The eccentricity is used to discriminate between the rather point-like object and passing background stars that appear as streaks. The largest structure with an eccentricity below 0.8 is assumed to be the object. Its centre of gravity is assumed to be the position of the object. Tests have shown that the much slower method of fitting a 2D normal distribution to the grey-scale image usually gives a rather similar central point.

The closed loop control tries to keep the object near a defined target point. This feature maintains the object into the field of view of the single photon detector used as receiver for the laser returns, which is placed in parallel to the camera. The detector covers an area of only $80 \mu \mathrm{m}$ (about 12 camera pixels) and cannot be moved mechanically.

A number of objects have been tracked for testing purposes. The tracking accuracy is evaluated by measuring the distance of the object to the camera target point (see figure 10). It has been found to depend mainly on the object distance (closer objects are harder to track) and on its position in the sky (positions close to the equatorial pole, or the polar star, are more difficult). Environmental parameters such as wind speeds or air turbulences also influence the tracking accuracy. 


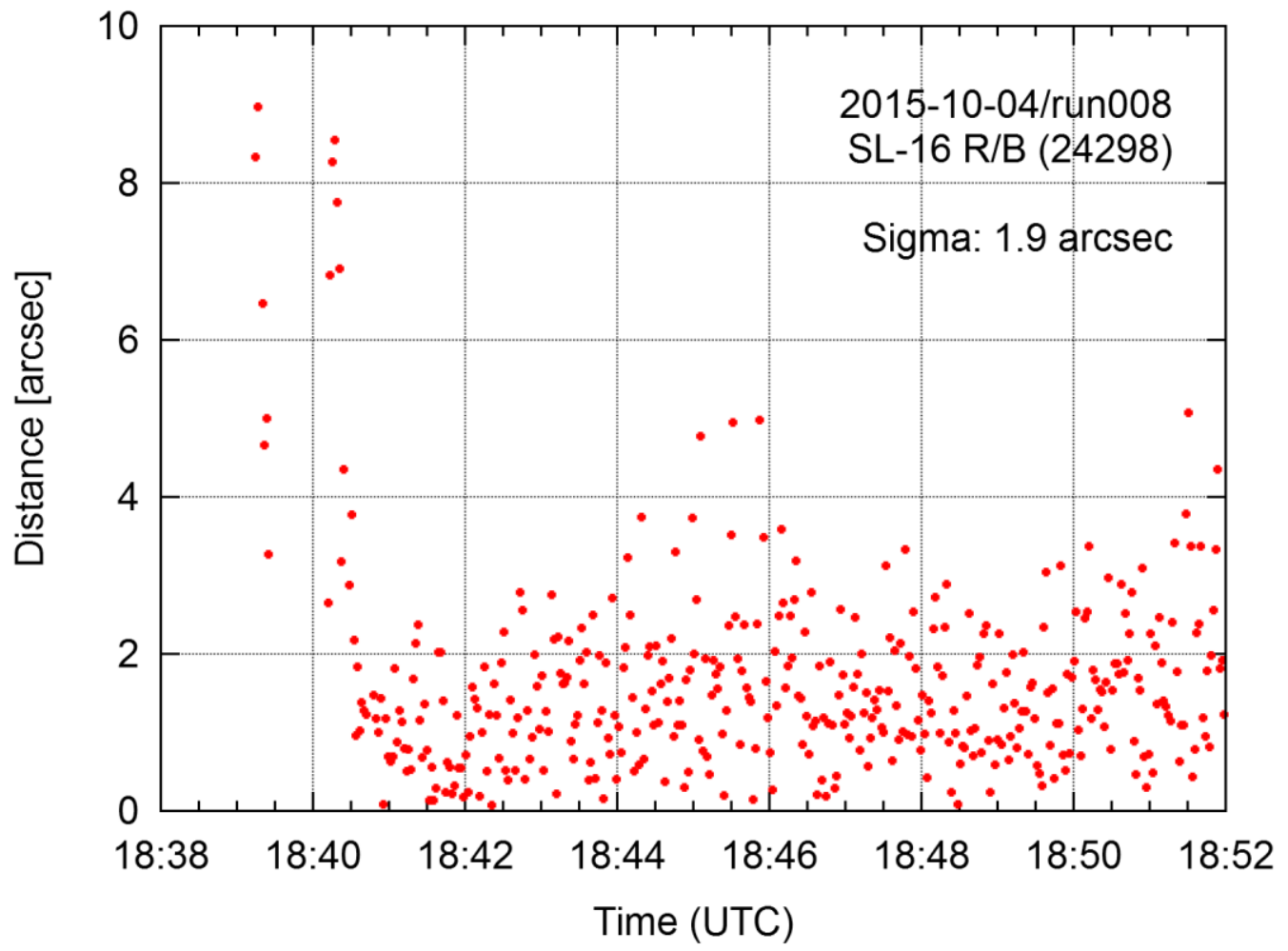

Figure 10: Deviation of object centre from camera target point throughout the observation (object 24298, tracked on October 4 th, 2015). During the pass, the object achieved an elevation angle of $42^{\circ}$ above horizon and a minimum distance of about $1180 \mathrm{~km}$.

A tracking RMS error of two arcseconds were achieved within 20 to 60 seconds after first acquisition for most objects, and it was shown to be maintained until the disappearance of the object at the horizon. However, depending on the factors mentioned above, tracking accuracy will sometimes deteriorates from five to ten arcseconds. In a survey of about 20 LEO satellites with retro-reflectors, about 15 could be tracked without any issues. The others had RMS tracking errors higher than 2", or took very long to reach that goal. Some small satellites were not found due to their faint signature. New tracking algorithms based on laser triangulation are being currently tested.

\subsection{Link budget Evaluation}

For link budget measurements a Nd:YAG laser operated at the fundamental wavelength (1064nm) with a maximal pulse energy of $300 \mu \mathrm{J}$, a FWHM pulse duration of $3 \mathrm{~ns}$ and a repetition rate adjustable between $1 \mathrm{kHz}$ and $10 \mathrm{kHz}$ is used. The laser is coupled into a multimode fiber which guides the light onto a laser transmitter directly attached to the tracking telescope. The laser transmitter consists of a beam steering unit enabling a fine steering of the beam with an accuracy of a few $\mu \mathrm{rad}$ over a range of about one mrad. The transmitted beam is expanded to about $6-8 \mathrm{~cm}$ at the transmitter output.

For the link budget evaluation, the laser transmitter was directed toward satellites equipped with bare retro-reflectors. After propagation through the atmosphere and reflection at the retro reflector equipped target a small part of the infrared light (at the single photon level) is collected by the tracking telescope. A specially designed beam splitter separates the infrared photons from the visible light used for tracking. The infrared light passes a narrow band pass filter with a 
bandwidth of $1 \mathrm{~nm}$ for noise reduction and is focused onto an InGaAs based SPAD (single photon avalanche diode) with detection efficiency up to $30 \%$ and a dark count rate below $2 \mathrm{kHz}$.

A dedicated electronic system including an event timer and a GPS receiver for synchronization to UTC is used to measure the time of flight (ToF) of the laser pulse [9]. The differences between the measured and expected ToF as given by the TLE data is computed. This enables the separation of signal events from the noisy background with a post processing algorithm and then extract the experimental return rate.

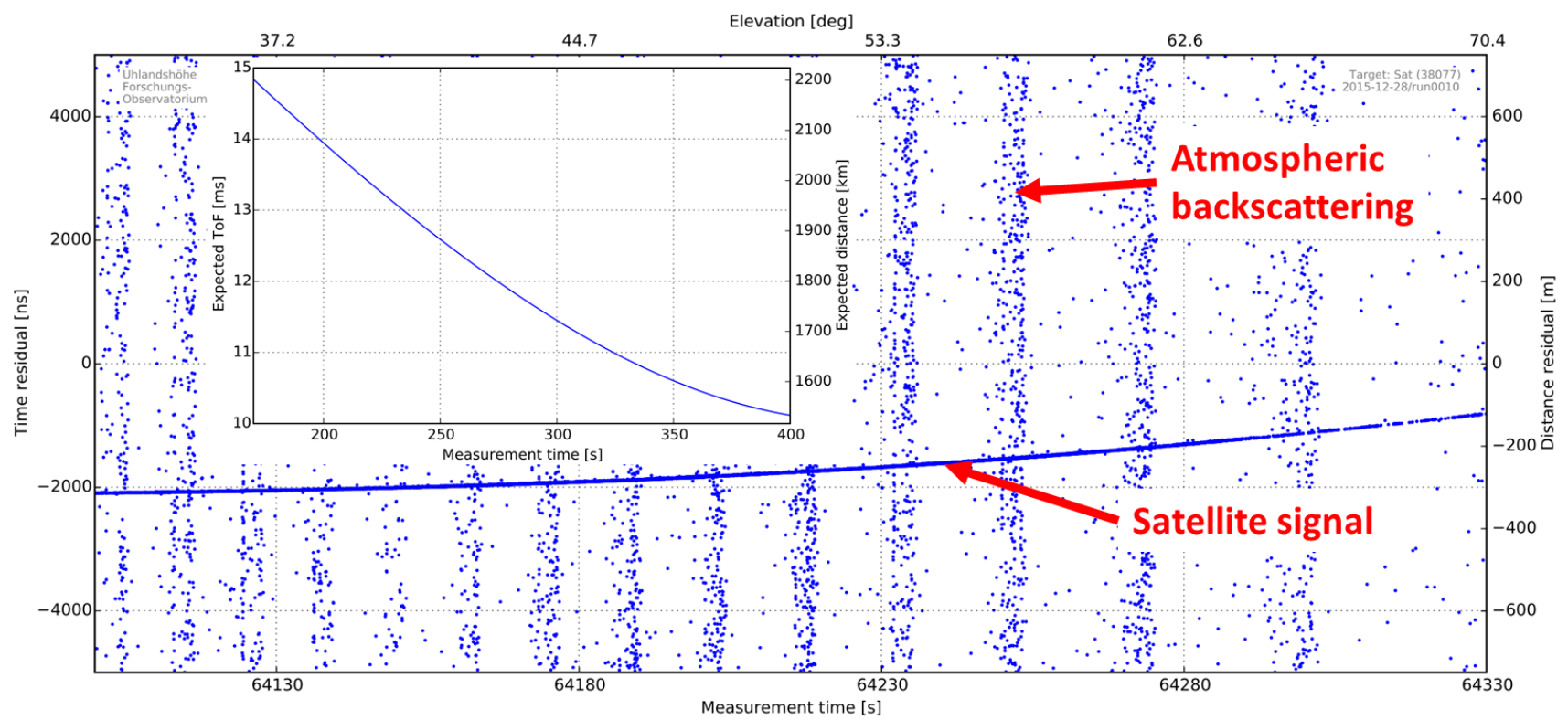

Figure 11: Residual plot of satellite LARES measured at 28.12.2015. Return from the satellite are clearly visible as line.

Figure 11 shows the remaining residuals (measured ToF minus predicted ToF) vs. time of satellite LARES measured at 28.12.2015. The predicted ToF was calculated from TLE data. The return events from the satellite are clearly as line whereas the noise events are randomly distributed over time. So called "pulse collision is visible as vertical bars. In this case, photons from a laser pulse propagating in the atmosphere are scattered into the detector while a returning pulse is expected. First return could be received at an elevation of $10-20^{\circ}$ and the average link settle time is about $30 \mathrm{~s}$ to $1 \mathrm{mn}$.

For calculation of the theoretical return rate the link budget equation as presented in reference [10] can be used for estimating the expected number of generated photo electrons per pulse. With the known (Poisson) detection statistics, the expected return rate can be calculated and compared to the experimental rate. The experimental link loss could be derived and estimated to be around $-150 \mathrm{~dB}$. This high link loss is mainly caused by the rather large divergence of the laser beam of about $250 \mu \mathrm{rad}$ half angle. Furthermore, the laser beam pointing was not optimal during the experiments. Thus, the link loss could be improved to about $-100 \mathrm{~dB}$ with a certain engineering effort.

\section{SECOND EXPERIMENT: LONG-RANGE DATALINK}

Experiment 2 targets the implementation of a 1Gbps data link between an UAV and a BS using a MRR. The MRR consists of an optical system and the EAM developed by ACREO. The link range will be at least $1 \mathrm{~km}$. 
A static preliminary test has been performed to show the high data rate capabilities of the developed system in an outdoors environment affected by turbulences. Figure 12 shows the setup. A $20 \mathrm{~mm}$ aperture MRR was setup on a tripod $200 \mathrm{~m}$ away from the base station.

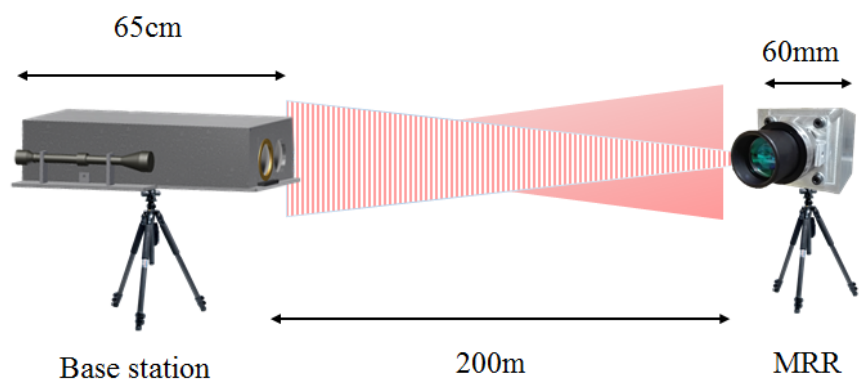

Figure 12: Data link demonstration set-up.

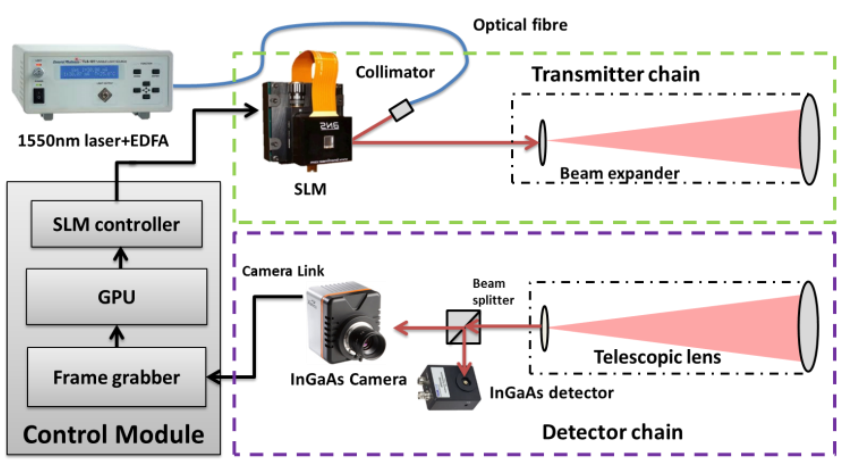

Figure 13: Base station optical module

Figure 13 shows the base station optical module. The system is based on a holographic beam steering module and an InGaAs camera acting as a position sensing device. It is a bi-static design, i.e. the transmitter and receiver do not share the same aperture. The transmitter chain uses a laser source, followed by a spatial light modulator (SLM) which implements beam steering and beam divergence control. After the SLM, an optical beam expander creates an output beam approximately $8 \mathrm{~cm}$ in diameter. The receiver consists of an $8 \mathrm{~cm}$ aperture telescopic optical system and a beam splitter that allows the light to be detected by both an InGaAs camera for tracking purposes and a photodetector for the data link. Further detail of the BS architecture is provided in [11].

A $15 \mathrm{dBm} 1549.5 \mathrm{~nm} \mathrm{CW}$ laser beam was used as the interrogating beam. A reliable data link of 150Mbps was achieved. The details of the experiment and the associated results are given elsewhere [12]. 


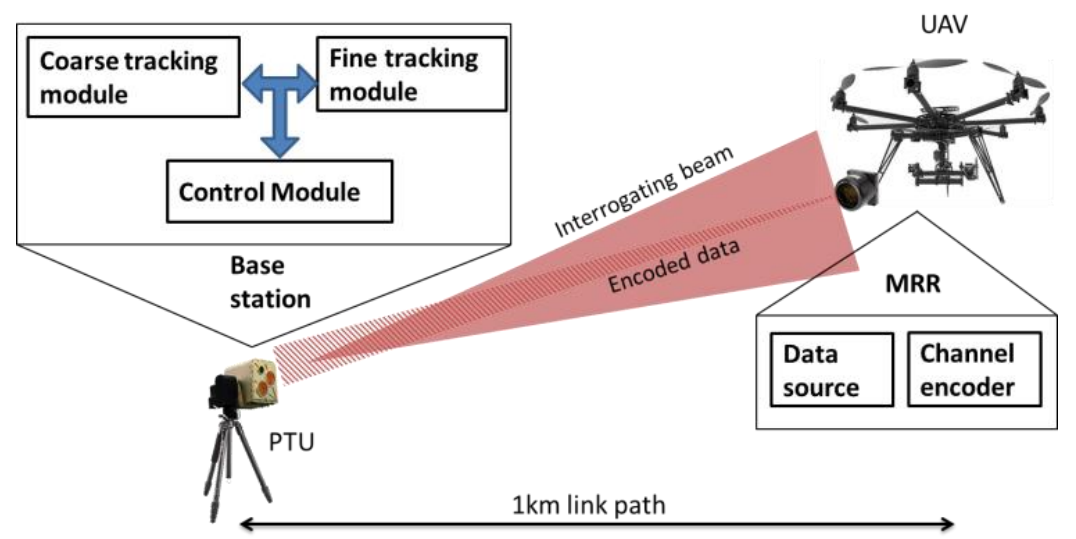

Figure 14: Experiment 2 system description

Figure 14 shows the architecture of the final experiment A high power Continuous Wave (CW) laser beam will be launched from the BS towards a UAV equipped with the MRR. The UAV position will be tracked using both coarse and fine tracking modules working cooperatively. The latter offers a high pointing accuracy within a limited narrow field of view (FOV) of $\pm 0.3^{\circ}$. The system FOV is significantly increased by using a Pan and Tilt Unit (PTU) based coarse tracking subsystem, which mechanically points the BS towards the UAV.

\section{CONCLUSIONS}

C3PO aims to show that communications to LEO satellites using MRR based transceivers is feasible. The project focuses on two experiments to demonstrate the feasibility of three critical components of a MRR based laser communication system i.e. the tracking, the link budget and the development and test of a MRR. So far, the tracking set-up is shown as capable of providing a 2 arcsec accuracy through a whole pass which is regarded as sufficient for the current state of the art pointing accuracy of nanosatellites [13]. A Link loss of 150db has been measured in the link budget experiment. Engineering effort on the ground station, especially for optimizing the laser beam divergence and refining the tracking performance would probably allow to reach a link loss of $100 \mathrm{~dB}$ necessary to achieve the targeted 1 Gbps data rate.

he MRR has been implemented and tested outdoors on a tripod. A data link of 150Mbps has been achieved at a range of 200m. A second design of MRR driver electronics is currently under development. They are expected to remove distortions introduced by the first design and then offer $1 \mathrm{Gbps}$ data rates capabilities. 


\section{REFERENCES}

[1] Emily Clements, Raichelle Aniceto, Derek Barnes, David Caplan, James Clark, Iñigo del Portillo, Christian Haughwout, Maxim Khatsenko, Ryan Kingsbury, Myron Lee, Rachel Morgan, Jonathan Twichell, Kathleen Riesing, Hyosang Yoon, Caleb Ziegler, Kerri Cahoy, "Nanosatellite optical downlink experiment: design, simulation, and prototyping," Opt. Eng. 55(11), 111610 (2016)

[2] L-3com.com, CTX-886 and CTK-830 datasheets

[3] gdmissionsystems.com; HRT150 datasheet

[4] sstl.co.uk, XTx400 datasheet

[5] R. Fields, D. Kozlowski, H. Yura, R.Wong, J. Wicker and C. Lunde, M. Gregory, B. Wandernoth, F. Heine, "5.625 Gbps bidirectional laser communications measurements between the NFIRE satellite and an optical ground station", 2011 International Conference on Space Optical Systems and Applications, 978-1-4244-9685$3 / 11$

[6] Christopher Schmidt, "OSIRIS Update and Outlook", OLEODL Workshop, 10.11.2016

[7] R.P. Welle ; S. Janson, D. Rowen, T. Rose, "CubeSat scale laser communications", 31st space symposium, Colorado USA, 2015

[8] Wang, Q., Noharet, B., Junique, S., Almqvist, S., Ågren, D. and Andersson, J. Y., "1550 nm transmissive/reflective surface normal electroabsorption modulator arrays", Electronics Letters, Volume 42, Issue 1., (2006)

[9] Humbert, Leif, et al. "Innovative laser ranging station for orbit determination of LEO objects with a fiber-based laser transmitter." CEAS Space Journal (2015): 1-5.

[10]Degnan, John J. "Millimeter accuracy satellite laser ranging: a review." Contributions of space geodesy to geodynamics: technology (1993): 133-162.

[11]C. Quintana, G. Erry, A. Gomez, Y. Thueux, G. E. Faulkner, and D. C. O'Brien, "Design of a holographic tracking module for long-range retro-reflector free-space systems," Appl. Opt., vol. 55, pp. 7173-7178, 2016.

[12] C. Quintana, Q. Wang, D. Jakonis, X. Piao, G. Erry, D. Platt, Y. Thueux, A. Gomez, G. Faulkner, H., Chun, M. Salter, and D. O'Brien Member, "High Speed Electro-absorption Modulator for Long Range Retroreflective Free Space Optics", IEEE Photonics Technology Letters, submitted December 15, 2016

[13]R. W. Kingsbury, "Optical communications for small satellites", PhD, Thesis, Massachusetts Institute of Technology (2015). 